SLAC-PUB-9010

October 2001

FERMILAB-Conf-01/139-T

\title{
Physics Goals for the Planned Next Linear Collider Engineering Test Facility
}

\author{
C. Bohn et al.
}

Contributed to the IEEE Particle Accelerator Conference (PAC 2001), 6/18/2001-6/22/2001, Chicago, IL, USA 


\title{
PHYSICS GOALS FOR THE PLANNED NEXT LINEAR COLLIDER ENGINEERING TEST FACILITY*
}

\author{
C. Bohn, L. Michelotti, J.-F. Ostiguy, M. Syphers, FNAL, Batavia, IL 60510, USA \\ H. Bluem, A. Todd, AES, Inc., Princeton, NJ 08543, USA \\ W. Gai, J. Power, J. Simpson, ANL, Argonne, IL 60439, USA \\ T. Raubenheimer, SLAC, Stanford, CA 94309, USA
}

\begin{abstract}
The Next Linear Collider (NLC) Collaboration is planning to construct an Engineering Test Facility (ETF) at Fermilab. As presently envisioned, the ETF would comprise a fundamental unit of the NLC main linac to include X-band klystrons and modulators, a delay-line power-distribution system (DLDS), and NLC accelerating structures that serve as loads. The principal purpose of the ETF is to validate stable operation of the powerdistribution system, first without beam, then with a beam having the NLC pulse structure. This paper concerns the possibility of configuring and using the ETF to accelerate beam with an NLC pulse structure, as well as of doing experiments to measure beam-induced wakefields in the rf structures and their influence back on the beam.
\end{abstract}

\section{INTRODUCTION}

The Engineering Test Facility (ETF), pictured in Fig. 1, is planned as the prototype of a fundamental unit of the NLC main linacs. The bimodal DLDS takes the power delivered by an 8-pack of 75 MW klystrons over a 3,168 ns pulse, divides it into $8510 \mathrm{MW}, 396 \mathrm{~ns}$ pulses, and delivers these high-power pulses to 8 sets of rf structures in phase with the arrival of the beam. Each pulse is long enough to span the fill time of the structure and accelerate the entire $265 \mathrm{~ns}$ length of the NLC bunch train.

Plans for the ETF are first to test the efficacy of the DLDS in transporting high rf power by way of two distinct modes, second to test its stability when the rf accelerating cavities are in place, and third to test its stability when loaded by an NLC-style bunch train. An ancillary goal is to use the ETF to do experiments on accelerator physics of relevance to linear colliders.

In what follows, we describe prospects for ETF regarding its operation with beam and its potential use for doing interesting accelerator-physics experiments.

\section{BEAM-INDUCED WAKES}

A fundamental concern for the NLC is the beaminduced rf wake. The wake acts back on the beam and degrades its quality both longitudinally and transversely. Various means of wake suppression are available; however, more confidence about their applicability is warranted prior to full-scale construction of the NLC main linacs. The main accelerator-physics goal for ETF is to accelerate cleanly a NLC-style bunch train. Auxiliary

*Work supported by Universities Research Association, Inc., under contract DE-AC02-76CH00300 with the U.S. Department of Energy.

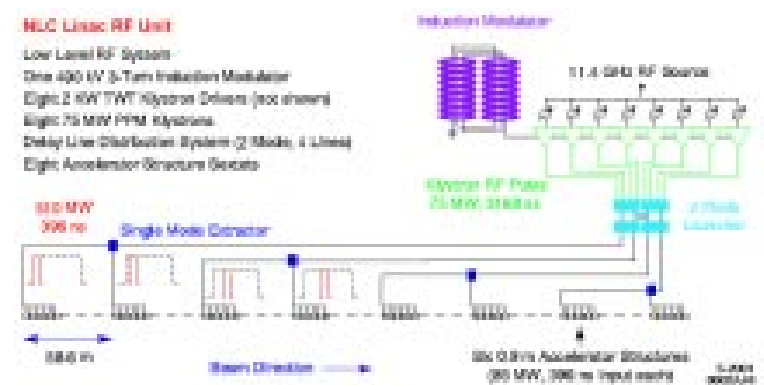

Figure 1: ETF minus injector and transport magnets.

goals are, where possible, to validate emittance-growth models, and to develop and test NLC beam diagnostics and instrumentation, as well as beam-based-alignment hardware and control algorithms.

ETF beam specifications that support the physics goals, and of which there is reasonable prospect of reaching [1], are listed in Table 1. Implied in the first entry is the ability to make a flat beam by adjusting the ratio of transverse emittances of the injected bunches [2]. Because the NLC main linacs will operate with flat beam, wake excitation versus bunch geometry is a relevant topic.

Table 1. ETF Beam Specifications

\begin{tabular}{|l|l|}
\hline Parameter & Specification \\
\hline injected rms bunch radius & $\begin{array}{l}\sigma_{r} \leq 0.5 \mathrm{~mm} \text { (for flat beam: } \\
\left.\sigma_{x} \sim 1.5 \mathrm{~mm}, \sigma_{y} \sim 0.15 \mathrm{~mm}\right)\end{array}$ \\
\hline injected rms bunch length & $\sigma_{z} \leq 0.5 \mathrm{~mm}$ (adjustable) \\
\hline rms normalized emittance & $\varepsilon_{n} \leq 10 \mu \mathrm{m}$ (at injection) \\
\hline $\begin{array}{l}\text { injected beam kinetic } \\
\text { energy }\end{array}$ & $\varepsilon_{i} \geq 50 \mathrm{MeV}$ \\
\hline $\begin{array}{l}\text { kinetic-energy spread } \\
\text { (single- \& multi-bunch) }\end{array}$ & $\Delta \delta E \leq 0.2 \%$ \\
\hline bunch charge & $q \leq 4 \mathrm{nC}$ (adjustable) \\
\hline $\begin{array}{l}\text { bunch train ("macropulse" } \\
\text { format) }\end{array}$ & $\begin{array}{l}1-\text { to- } 95 \mathrm{bunches} \text { separated } \\
\text { by } 2.8 \mathrm{~ns}\end{array}$ \\
\hline $\begin{array}{l}\text { output beam kinetic } \\
\text { energy }\end{array}$ & $\varepsilon_{f} \geq 2 \mathrm{GeV}$ \\
\hline
\end{tabular}

To assess the ETF's potential to support cumulative beam-breakup (BBU) experiments, we developed analytic BBU estimates by modeling it as a smooth, continuous linac per the parameters of Table 2. The $4 \mathrm{nC}$ bunch charge and $2 \cdot 10^{15} \mathrm{~V} / \mathrm{C} / \mathrm{m}^{2}$ long-range wake amplitude are postulated to support parametric studies of multibunch BBU. By contrast, the nominal NLC bunch charge is 1.25 $\mathrm{nC}$ with $1.4 \mathrm{~ns}$ spacing. In addition the long-range wake 
amplitude is ideally $\sim 0.2 \cdot 10^{15} \mathrm{~V} / \mathrm{C} / \mathrm{m}^{2}$ in a rounded, damped, detuned structure (RDDS), an order of magnitude smaller than that assumed in Table 2. We presume the ETF will comprise 48 RDDSs, each with adjustable wake amplitude.

Table 2: Model ETF Linac Parameters for BBU Estimates

\begin{tabular}{|l|l|}
\hline Parameter & Value \\
\hline short-range wake amplitude & $10^{17} \mathrm{~V} / \mathrm{C} / \mathrm{m}^{2}$ \\
\hline long-range wake amplitude & $2 \cdot 10^{15} \mathrm{~V} / \mathrm{C}^{2}{ }^{2}$ \\
\hline bunch charge & $4 \mathrm{nC}$ \\
\hline injected beam kinetic energy & $0.05 \mathrm{GeV}$ \\
\hline output beam kinetic energy & $2 \mathrm{GeV}$ \\
\hline deflecting-mode frequency & $14.95 \mathrm{GHz}$ \\
\hline $\begin{array}{l}\text { bunch train ("macropulse" } \\
\text { format) }\end{array}$ & $\begin{array}{l}90 \mathrm{bunches} \text { separated } \\
\text { by } 2.8 \mathrm{~ns}\end{array}$ \\
\hline linac length & $60 \mathrm{~m}$ \\
\hline number of betatron periods & 6 \\
\hline
\end{tabular}

The longitudinal short-range wake is believed to be of little concern in the NLC. Wake calculations and NLC simulations done to date at SLAC suggest this wake becomes important only for very short bunches. For example, a $0.1 \mathrm{~mm}, 2 \mathrm{nC}$ bunch would suffer $\sim 1 \%$ energy spread, and this can be compensated by accelerating the beam $\sim 10^{\circ}$ off crest. For a bunch length of $\sim 0.5 \mathrm{~mm}$, the single-bunch energy spread may be too small to observe in ETF, but it will be of interest to confirm this.

Plans are to compensate the longitudinal long-range wake in the NLC by shaping the rf-pulse amplitude (and phase, if necessary), as was successfully demonstrated at NLCTA [3]. The ETF would extend the NLCTA studies to higher beam loading from an NLC-style bunch train, thereby accessing higher-order modes (HOMs) that may, e.g., be in resonance with the bunch spacing and potentially cause a few-percent energy spread along the bunch train. This cannot be done at NLCTA because its beam fills every rf bucket at $11.424 \mathrm{GHz}$. A potential flaw is that HOMs are sensitive to details of the structure design so that an ETF experiment will likely not replicate precisely the NLC dynamics. However, successfully demonstrating high-frequency-wake compensation under "arbitrary" conditions will lend confidence to the technique's applicability in the NLC proper.

The transverse short-range wake drives cumulative single-bunch BBU, potentially leading to strong emittance dilution in the NLC. Plans are to counter it with accurate alignment and BNS damping. It also is potentially severe in the ETF, wherein it imposes a constraint on the bunch length. Results of an analytic calculation [4] of the singlebunch BBU envelope for the model ETF linac of Table 2 indicates that a $0.5-\mathrm{mm}$ rms bunch length will give rise to a transverse head-to-tail displacement comparable to the initial displacement. If the initial displacement is sufficiently small, e.g., $0.1 \mathrm{~mm}$, the projected transverse image of the bunch at the end of the linac will likewise be correspondingly small. However, single-bunch BBU can be produced in the ETF by misaligning the beam and/or various accelerator components. Its magnitude is also sensitive to the short-range wake amplitude, and singlebunch BBU therefore provides a diagnostic of the performance of the rf structures. Unfortunately, the ETF would necessitate a large energy spread across the bunch, some tens of percent, to suppress the wake, and so it probably cannot be used to study this method of compensation. By contrast, the main NLC linacs permit suppression with a percent-level energy spread [5].

The transverse long-range wake drives cumulative multibunch BBU and is another concern as a source of emittance dilution in the NLC. Plans are to counter it with detuned rf structures and, if needed, a focusing variation or energy spread along the bunch train. However, just as for the single-bunch case, the ETF likewise necessitates a large energy spread across the bunch train, some tens of percent, to suppress multibunch $\mathrm{BBU}$, and it probably cannot be used to study such compensation. In the NLC, given no resonant coupling between the beam and the transverse long-range wake, a percent-level energy spread would suffice [6].

Derived from the model ETF linac of Table 2, Fig. 2 shows the possible outcome of an experiment designed purposely to generate multibunch BBU. The head-to-tail offset of the bunch train is some 20 times that of a single bunch. The circles in Fig. 2 represent the bunch locations at the ETF exit as calculated numerically from the equation of transverse motion, and the solid curve is the analytic solution for the envelope. The key point is that there is a reasonable prospect of doing multibunch BBU experiments with ETF, provided the structures' long-range wake amplitude can be adjusted to high values.

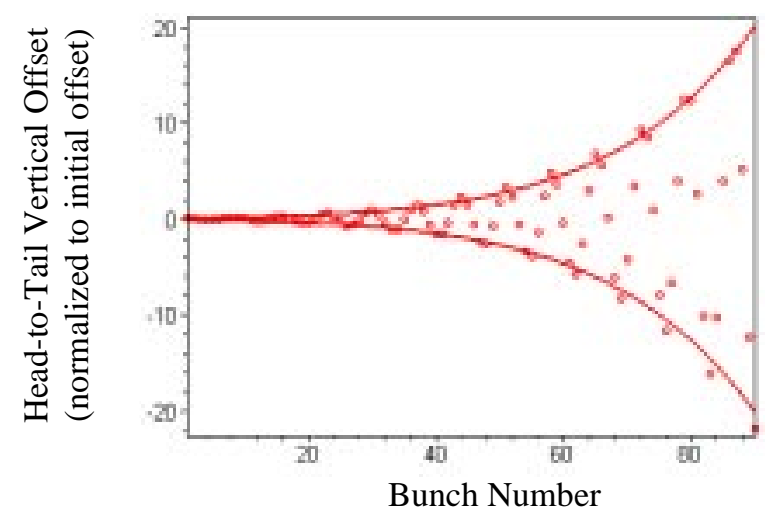

Figure 2. Estimated capacity for generating multibunch BBU in ETF. The envelope is generated analytically.

\section{BEAM SIMULATIONS}

The RDDS structures are designed to accelerate a highenergy electron beam (>10 GeV). Mitigation of the longrange wake is based on dipole-mode detuning presuming the beam travels in a straight line without significant disruption. Here, via LIAR simulation with the strawman lattice of Fig. 3, we investigate the feasibility of injecting a $50 \mathrm{MeV}$ beam from a low-emittance rf gun 
directly into the ETF. The lattice is divided into 8 supercells delimited in Fig. 3 by thick vertical lines. All supercells have at their center a girder with 6 consecutive 0.9-m RDDSs, and FODO cells occupy the rest of the space. In the first two supercells, the structures are interleaved with the quadrupoles to match the low-energy injected beam to the ETF. The beta functions settle into a regular periodic pattern beyond the second supercell.

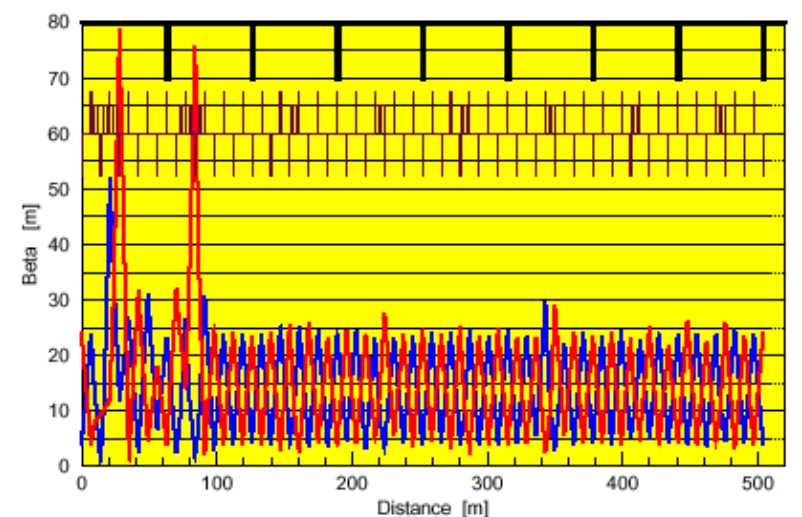

Figure 3. ETF horizontal (blue), vertical (red) $\beta$-functions.

The simulation incorporates rf structures devoid of manufacturing errors; the longitudinal wake is that of the RDDS, and the transverse wake is that of the DDS, somewhat worse than RDDS. LIAR treats structures as thin elements. For a more realistic treatment we partitioned each RDDS into imaginary substructures and tailored the dipole-mode frequency distribution in each to map onto its respective portion of the gaussian distribution characterizing the entire structure. The results are nearly identical with and without partitioning.

Figure 4 shows the projected transverse rms normalized emittance of a train of $4 \mathrm{nC}$ bunches injected at $0.5-\mathrm{mm}$ offset as it passes down the ETF. The bunch centroids stay near the beamline axis; multibunch BBU is negligible. The results highlight the feasibility of injecting a $50 \mathrm{MeV}$ beam into the ETF and accelerating it without catastrophic degradation. The injector of Ref. [1] should reach $\sim 190 \mathrm{MeV}$, an easier energy to match.

\section{WAKE MEASUREMENTS}

A high-resolution system based on the drive-witness scheme [7] is being considered for direct measurement of beam-induced wakes. The high-energy beam from the ETF linac (drive beam) passes through a device under test (DUT) to excite a wake. Its trajectory through the DUT can be varied either by changing the upstream beam optics or by mounting the structure on a moveable stage. A variable time $(0-10 \mathrm{~ns})$ later a low-energy witness beam is sent to probe the wake. The witness beam is kept on the same trajectory as the drive beam by placing a corrector magnet over the DUT such that its magnetic field cancels the deflection from the transverse wake. The measured transverse wake is proportional to the magnetic field of the corrector. Using a low-energy beam as the probe makes this scheme very sensitive because the wake

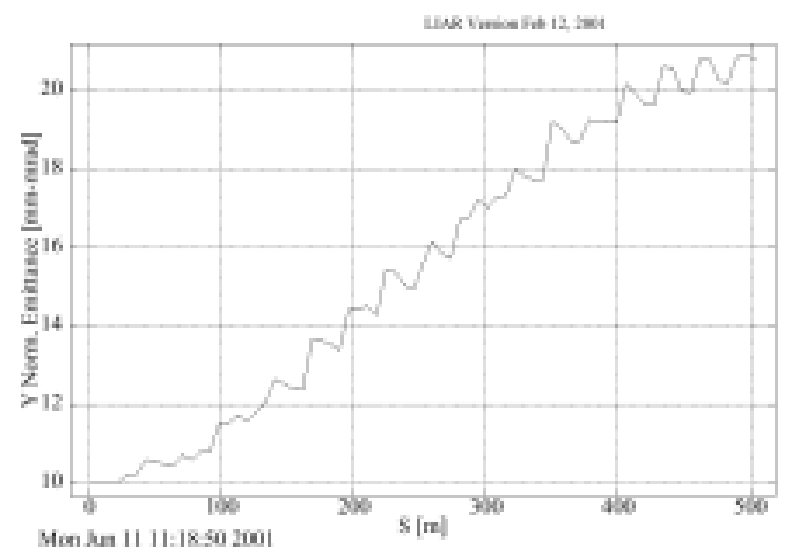

Figure 4. Vertical emittance of the bunch train (in $\mu \mathrm{m}$ ) plotted along the ETF. The horizontal emittance is lower.

can more easily deflect the beam. As the drive beam emerges from the DUT, a chicane steers it to other downstream experiments. It also steers the witness beam into a separate beamline containing an energy spectrometer and several BPMs for measuring the longitudinal and transverse wakes, respectively.

Given a BPM with $\sim 1-\mu \mathrm{m}$ least-count offset sensitivity and a $20 \mathrm{MeV}$ witness beam, the minimum detectable deflection energy is $\sim 2 \mathrm{eV}$. The corresponding resolution is $\sim 1 \mathrm{TeV} / \mathrm{C} / \mathrm{m}^{2}$, some 50 times better than ASSET. It should be high enough to isolate the contributions to the deflection from individual modes of different azimuthal symmetries (dipole, quadrupole, etc.). Table 3 compares the projected ETF capabilities against those of ASSET. An existence proof of the beamline for this system has been completed [8], and it shows that a satisfactory solution can be obtained with relatively simple optics.

Table 3. Proposed ETF vs. Present ASSET Capabilities

\begin{tabular}{|l|l|l|}
\hline Parameter & ETF & ASSET \\
\hline drive energy & $2 \mathrm{GeV}$ & $1.19 \mathrm{GeV}$ \\
\hline drive charge & $1-4 \mathrm{nC}$ & $3 \mathrm{nC}$ \\
\hline witness energy & $20-50 \mathrm{MeV}$ & $1.19 \mathrm{GeV}$ \\
\hline max beam offset & $\sim 4 \mathrm{~mm}$ & $4 \mathrm{~mm}$ \\
\hline wake resolution & $1-5 \mathrm{TeV} / \mathrm{C} / \mathrm{m}^{2}$ & $50 \mathrm{TeV} / \mathrm{C} / \mathrm{m}^{2}$ \\
\hline measured wakes & at least quadrupole & dipole \\
\hline drift length & $10 \mathrm{~m}$ & $3 \mathrm{~m}$ \\
\hline BPM resolution & $\sim 1 \mu \mathrm{m}$ & $\sim 3 \mu \mathrm{m}$ \\
\hline
\end{tabular}

\section{REFERENCES}

[1] A. Todd, H. Bluem, and C. Bohn, these Proceedings.

[2] D. Edwards, et. al., Proc. Linac2000, SLAC-R-561, 122 (2000).

[3] R.D. Ruth, et. al., Proc. PAC97, IEEE Cat. No. 97CH36167, pp. 439-443 (1998).

[4] C.L. Bohn, J.R. Delayen, PRA 45, 5964 (1992).

[5] G. Stupakov, SLAC Report No. LCC-0012 (1999).

[6] G. Stupakov, SLAC Report No. LCC-0027 (1999).

[7] H. Figueroa, et. al., PRL, 60, 2144 (1988).

[8] J. Simpson, ANL-HEP-WF-204 (2001). 